\title{
Asymmetric Cyclization of Ene-Allenes by Nickel Catalysis
}

General reaction:
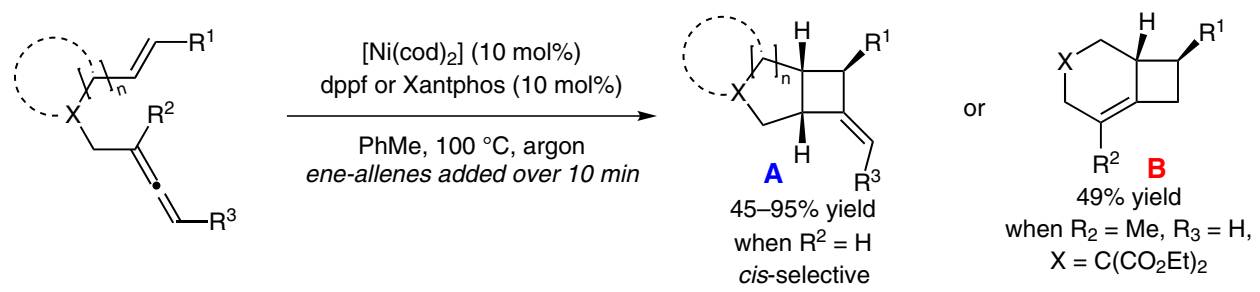

\section{Selected examples:}

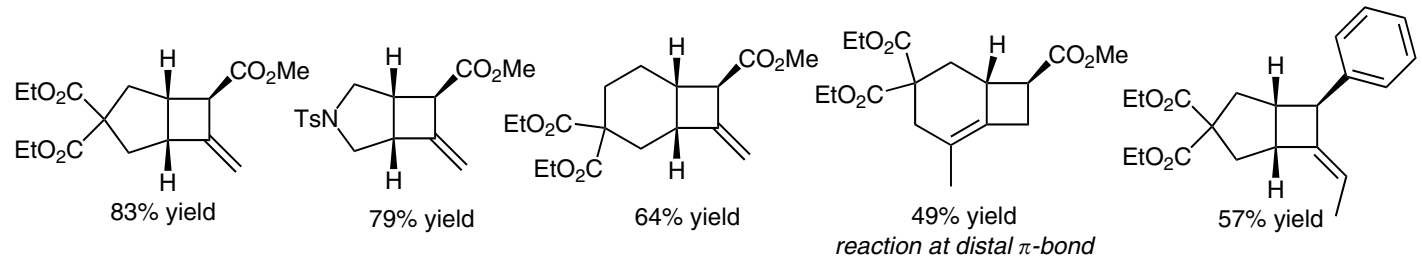

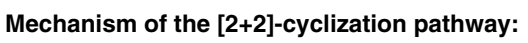

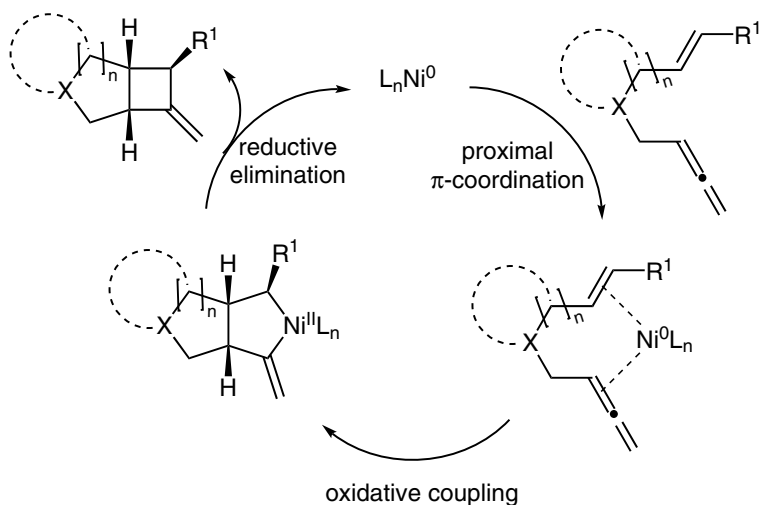

Significance: The [2+2] cycloaddition of ene-allenes is a powerful methodology in the construction of fused cyclobutanes. However, one of the challenges of known protocols is the limited substrate scope, tolerating only styrene or diene $\pi$ components. Alexanian and Noucti demonstrate the utility of nickel catalysis in a stereoselective [2+2]-cycloaddition to generate diverse carbocyclic frameworks $\mathbf{A}$.

sYNFACTS Contributors: Mark Lautens, Charles C. J. Loh Synfacts 2015, 11(6), 0613 Published online: 18.05.2015 DOI: 10.1055/s-0034-1380770; Reg-No.: L04715SF
Comment: It is noteworthy to point out the effectiveness of this protocol to cyclize terminal allenes which are not tolerated in other known methods. Moreover, an interesting observation was discovered where methyl substitution at $\mathrm{R}^{2}$ changes the $[2+2]$ cycloaddition from the proximal to the distal $\pi$-bond of the allene, generating the bicyclo[4.2.2] scaffold $\mathbf{B}$ instead. 